\title{
Injury risk and prevention in context
}

\section{Roderick J McClure}

\begin{abstract}
And when all the tigers are caged, what then the need to hide, the world all being safe? ${ }^{12}$
\end{abstract}

There is substantial need indeed if the results of the study by Pickett et $a l^{3}$ reported in their article published in this issue of Injury Prevention (see page 376) are an indication. While the reported study is one of the most important recently undertaken, it is not only in the analytical results that the importance of this paper lies, but also in the questions the authors raise. The study was grounded in the ecological understanding of the causation and prevention of injury introduced by Haddon in his seminal paper published 40 years ago. ${ }^{2}$ Pickett et al have worked towards actualising that theory (using multi-level models of causation), describing the concept of 'exposure' within an ecological framework, and accurately measuring the relevant contextual and individual level component causes. The issues addressed by the authors are critical (both in content and timing) for the future of injury prevention practice, and, in exploring these issues through empirical study rather than conceptual discussion, the authors have made an important contribution to the field.

The conventional position on injury prevention recommends that efforts should be restricted to a consideration of 'the necessary conditions for harmful results' and not of the 'complex mix of factors' that lead to serious injury events. ${ }^{45}$ Pickett et al have based their study on the alternative position; that it is exactly on this complex mix of factors that the success of population-based programmes depends. ${ }^{6-8}$ They suggest that improvements in the effectiveness of populationbased injury prevention programmes could be achieved if: (i) research were refocused on identifying the contexts in which proximal risk factors were situated; and (ii) interventions were designed to address both the proximal risk factors and the

Correspondence to Professor Rod McClure, Accident Research Centre, Room 115, Building 70, Monash University, Clayton, Victoria 3800, Australia; rod.mcclure@monash.edu context in which the proximal risk factors are situated.

It is hard to take objection with the claim for the importance of context as a determinant of injury risk. On the basis of the argument propounded by Rose in $1985,{ }^{9}$ it is a self evident conclusion from injury data, that an individual who moves from Sweden to Africa immediately changes his or her risk of road trauma by an order of magnitude simply by changing the contexts in which he or she drives. The problem is that while the concept of ecological causation of injury is well accepted, it has proved to be difficult to empirically quantify the relationships between components of the web of causation. There have been no studies that have successfully identified the contextual and individual level causal factors for a given injury type and then undertaken an injury prevention programme based on this information. ${ }^{10}$

Pickett et al note that efforts to ground research design and analysis on conceptual theory is a promising advance in the field of injury control. They attempt to actualise the ecological understanding of injury causation in terms of a mathematical model that allows two levels of variables (contextual and individual) where these two levels interact multiplicatively to determine injury risk. This analytical model is then tested using observed data obtained from a large-scale cohort study. In this study, the individual exposure used was duration that people were engaged in farm work, and the contextual exposures involved three variables covering the socioeconomic, physical farm work environment, and cultural farm work environments.

In the main analysis to confirm the hypothesised multi-level model, hours of farm work was strongly correlated with time to first injury but this relationship was not modified by the contextual environments. The study results found that of the contextual environments measured, only the physical work environment was directly associated with time to first injury. Thus the authors concluded that the data did not support an ecological model of injury causation.
Critical to the interpretation of these results is the difficult question of how to understand the term 'exposure' in the context of the ecological model characterised in the multilevel Cox proportional hazards analysis. In epidemiology, exposure time is usually defined in relation to duration of time exposed to a factor thought to be causally related to the outcome of interest. For this analysis however, the authors have defined individual exposure in relation to duration of time farming and then defined risk factors and risk environments as additional variables in the equation. The strong relationship found between duration of time farming and time to first injury independent of the other variables in the model suggests there were risk factors to which participants were exposed on the farm that were not defined within either the confounding or contextual variables included in the model. Thus, while the simple interpretation of the lack of an interaction effect between the individual exposure time and contextual variables might be taken as lack of support for the ecological model, an alternative interpretation is plausible.

The observed risks associated with duration of time farming could show that the risk of injury exists not only in specifically identifiable hazards and hazardous environments, but also when all known risk factors and risk environments are controlled. As alluded to in the opening sentence of this editorial, the study by Pickett et al appears to demonstrate that risk cannot simply be identified in discrete hazards or hazardous environments that can be caged to create 'risk free' zones where individuals are totally safe. ${ }^{11}$ Thus the results of the study appear to support the ecological model by showing that the risk of injury is a ubiquitous part of the human condition. While energy may be the ultimate agent for injury, it is also necessary for life itself.

An alternative interpretation of the null result is the much simpler (but harder to solve) problem of measurement error. It is difficult to measure proximal risk factors involved in an injury event, but measuring contextual risk factors is ever so much harder. Given the substantial number of possible risk factors, and their sometimes subtle and unknown nature, accurate measurement is a complex expectation. As the authors have identified, the factors selected to characterise the model may not have been the critically important components of the ecological model's structure. The validity and reliability of 
the measures used were unknown. The lack of observed associations between the contextual risk factors and time to first injury in Pickett et al, and the lack of any relationship between the levels of factors, may reflect a lack of precision and validity in measurement, rather than problems with the concepts being tested. Future work in this area may benefit from more appropriate measures of relevant context, more accurately measured. The study authors however rightly note that there have been few attempts previously to quantify these variables and relationships, and the contribution made by this study in identifying the challenges is an extremely useful contribution to knowledge.

For the ecological approach to move beyond rhetoric, injury prevention researchers need to identify and measure the relevant contextual factors responsible for the circumstances and events that can lead to injury, as well as the risk factors relating to the potential energy exchange process. Pickett et al have made important advances in thinking through the issues involved. If, as suggested by the results of the featured study, caging all tigers may not be possible, then a contextual approach may at least identify ways of being able to live with them in a manner that causes the least possible harm.

\section{Competing interests None.}

Contributors RM was the sole contributor to all aspects of the article's preparation.

Provenance and peer review Commissioned; not externally peer reviewed.

Published Online First 24 October 2010

Injury Prevention 2010;16:361-362.

doi:10.1136/ip.2010.028175

\section{REFERENCES}

1. Bolt R. A Man for all Seasons: A Play of Sir Thomas More. London: Heinemann, 1960.
2. Haddon W. On the escape of tigers: an ecologic note. Am J Public Health 1970;60:2229-34.

3. Pickett W, Hagel L, Day A, et al. Determinants of agricultural injury: a novel application of the population health theory. Inj Prev 2010;16:376-82

4. Robertson LS. Causal webs, preventive brooms, and housekeepers. Soc Sci Med 1998;46:53-8.

5. Robertson LS. Prevention of motor-vehicle deaths by changing vehicle factors. Inj Prev 2007:13:307-10.

6. Zwi A. Injury control in developing countries: context more than content is crucial. Inj Prev 1996;2:91-2.

7. Martuzzi M. Science, policy, and the protection of human health: a European perspective. Bioelectromagnetics 2005;17:S151-6.

8. Blank D. Injury control from the perspective contextual pediatrics. J Pediatr (Rio J) 2005: 81(5 Suppl):S123-36.

9. Rose G. Sick individuals and sick populations. Int $J$ Epidemiol 1985; 14:32-8.

10. McClure R, Davis E, Yorkston E, et al. Special issues in injury prevention research: developing the science of program implementation. Injury 2010;41(Suppl 1): S16-19.

11. Widome MD. Injury illiteracy. Pediatrics 1992;89:1091-3. 